\title{
Controlled image fusion
}

\section{ABSTRACT}

A Virtual Lab to experiment on controlled fusion of two digital images has been developed. The resulting image has user pre-defined percentages of each contributing image. With the aim on improving the quality of the resulting image in the fusion, the Virtual Lab has been enabled with the option of selecting a color to be ignored during the fusion process, in this way this color is not fussioned. Successful experiments have been executed fusing three images using the same algorithm.

Keywords: digital image processing, controlled image fusion, algorithms

Fusión CONTROLADA DE IMÁGENES

\section{RESUMEN}

Se ha desarrollado un laboratorio virtual para experimentar la fusión controlada de dos imágenes digitales. La imagen resultante posee porcentajes predefinidos de cada imagen participante. Con la finalidad de mejorar la calidad de la imagen resultante de la fusión, el laboratorio virtual incluye la opción de ignorar un color durante la fusión, lo que hace que este color no experimente fusión alguna. Se han realizado experimentos exitosos fusionando tres imágenes con el mismo algoritmo.

Palabras clave: procesamiento digital de imágenes, fusión controlada de imágenes, algoritmos

\section{INTRODUCTION}

The most important difference between human vision and machine (computer) vision is that "humans recognize" while "machines measure".

The objective of image processing $[1,5]$ in science and engineering applications is to extract useful information from the radiation emitted by objects. An imaging system gathers this radiation and renders it as an image. The radiation emitted by objects may be due to self-emission, induced emission, reflection, refraction, absorption, and scattering.

The most common types of radiations $[6,7]$ used to obtain images are:

- Electromagnetic radiation: Gamma rays, X-rays, Ultraviolet, Visible light, and Infrared.

- Particle radiation: Electrons and Neutrons.

- Acoustic radiation: Echography (the use of elastic waves to construct images).

Image Fusion [8-10] is a technique based on the blending of different images of a given scene into a single resulting image, which contains more useful information than any of the individual contributing images. The images taking part in the fusion may be obtained with different radiation types and by means of different techniques. As an example consider the fusion of two images, an $X$-ray image of a piece of luggage and its corresponding infrared image.

Obviously the resulting image has more information than that contained in any of the component images and consequently it is much more useful, in this case to the customs officers. Current technology has developed different techniques [7] to generate images, namely, computer tomography, ultrasound, nuclear magnetic resonance, etc. These techniques allow obtaining different images of a same object or scene. Image fusion also allows visualizing an image in the frame of reference of another.

Images are used since long ago in medicine, where traditionally $\mathrm{X}$-rays are used to visualize bones. Since relatively not long ago, other radiations are being used to obtain images of soft tissue. There might be situations where it is desired to have images of

Founding Director, VirtualDynamicsSoft: Science \& Engineering Virtual Labs. Physics faculty member at UNMSM. Director@VirtualDynamics.Org www.VirtualDynamicsSoft.com 

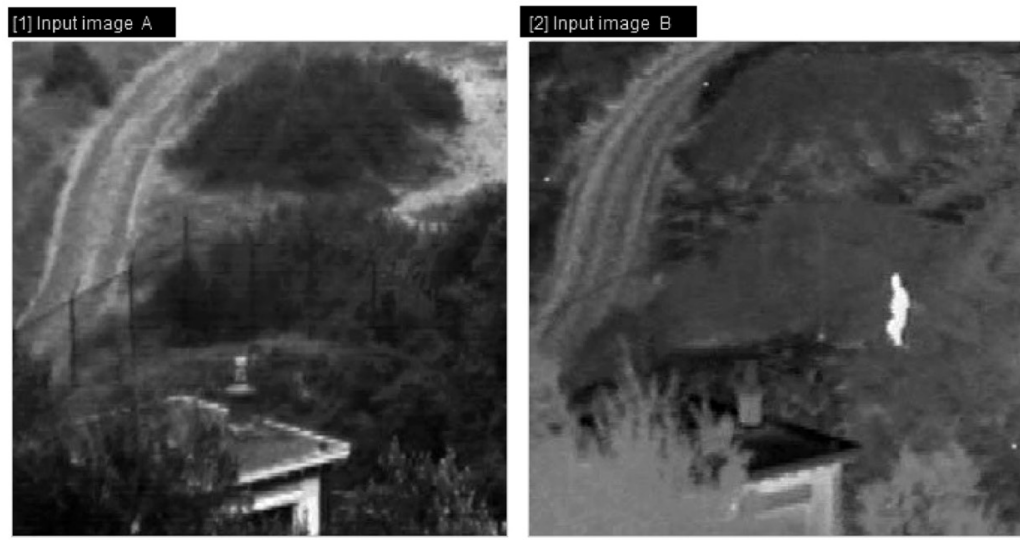

Fig 1

In the upper line two images of the same scene but obtained with different radiations are shown.

(A) Left: image in the visible spectrum.

(B) Right: infrared radiation.

Below: Three different cases of controlled fusion:
(1) $50 \%$ and $50 \%$
(2) $65 \%$ and $35 \%$
(3) $75 \%$ and $25 \%$
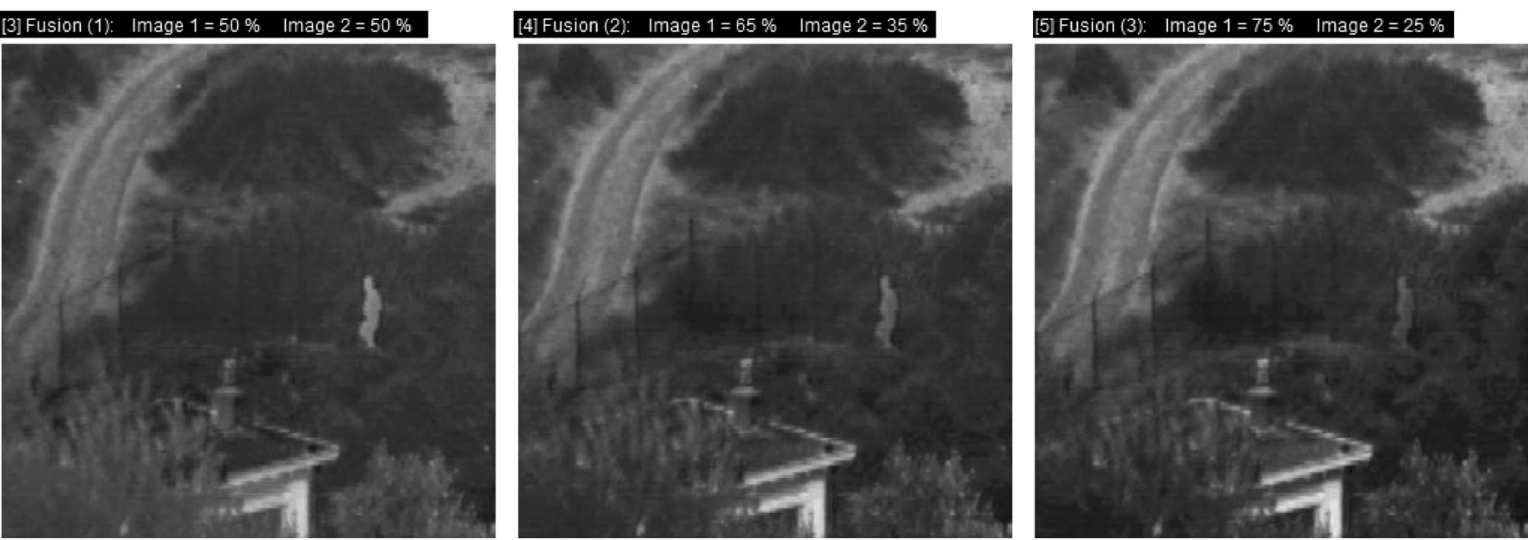

bones and soft tissue, in a single one. This is now possible with the fusion of images.

With respect to the organization of this paper, next, a panoramic view of image fusion and related themes is presented; this will be followed by a description of the Controlled Image Fusion algorithm, the results of the experiment and the conclusions.

\section{Applications of Image Fusion}

Image fusion technique [8-10] are being used in restoration of distorted images, as a diagnosis tool in some medical applications, in biometric applications, remote sensing, surveillance and security systems, defense systems, nondestructive evaluation, image quality assessment, etc.

\section{Sensors in image acquisition}

In many applications the image generated by a single sensor [10] cannot provide enough information about a scene. As an example consider the case of an infrared camera detecting a hot body in a scene, this information should be complemented by a visible light photograph of the same panorama so that the hot spot may be geometrically located in the picture. Ideally the two images must be fused in a single one. Some examples of sensors producing images are optical cameras, millimetre wave cameras, infrared cameras, x-ray imagers, and radar imagers.

\section{Alignment of images before fusion}

Since images to be fused are to be geometrically superimposed, the sensors used for image fusion need to be accurately aligned so that their images will be in spatial registration. If this is not possible, then registration algorithms are to be applied before image fusion [8] .

\section{Main drawback of Image Fusion}

There are many algorithms to achieve image fusion and the most common is the one based on pixel weighted average, but this has the undesired effect of suppressing highlighting image features, because it generates a low contrast image [8] with a rather colorless washed-out look. This is, the images resulting from the fusion exhibit a wear away appearance.

Researchers have tackled many attempts so as to subdue this negative outcome [8].

Notwithstanding image fusion has its disadvantages, since it achieves the combination of two different 


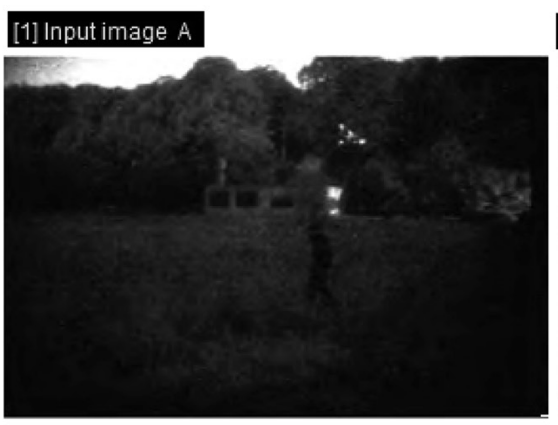

\section{[2] Input image $\mathrm{B}$}

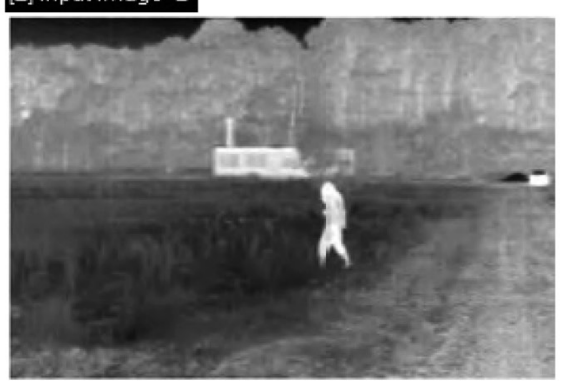

Fig 2

Top: Two input images $A$ and $B$ of the same scene, obtained with unknown radiations.

Below: (Output) three different cases of controlled fusion of $\mathrm{A}$ and $\mathrm{B}$
(1): $50 \%$ and $50 \%$
(2) and (3): $30 \%$ and $70 \%$

The difference between the resulting images (2) and (3) is that a dark color was ignored during fusion. It can be seen that ignoring a color during fusion improves the image
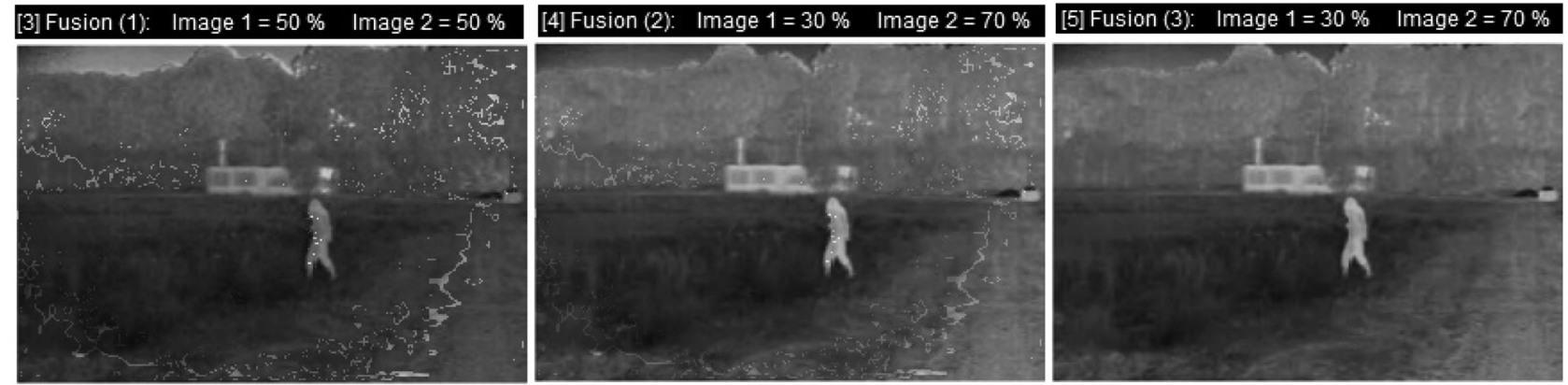

images, it becomes a very powerful image processing technique, with plenty of critical applications and hence very much research is being carried out on it [8$10]$. The technique of image fusion has proven to be very useful in medical and in remote sensing imaging.

\section{CONTROLLED IMAGE FUSION}

Controlled image fusion allows dosing the contribution of each participating image in the fusion process so that the resultant image contains predefined percentages of the component images. The merging of two or more images under this scheme operates on the complete area of the images and it generates a sort of chemical combination of them, precisely like molecules do in an actual chemical combination, this is in contrast with the thresholded [11-13] overlapping of images, where only those pixels above a pre-defined threshold are involucrate in the image merging.

\section{The algorithm for controlled image fusion}

From the many algorithms to achieve image fusion, the Weighted Average [8-10] of participating images is applied in the work being reported here.

In the Weighted Average each pixel $(x, y)$ of the image resulting from the fusion of two images contains -as the name of the algorithm suggests- the weighted average of pixels $(x, y)$ in the two images partaking in the process.
Programming these types of algorithms dealing with images is more an art than a science. Experienced programmers usually have completed a tool box containing software tools developed by themselves, where for optimization and portability purposes most of the developed routines are in Assembler language and usually in order to control memory consumption by these tools, some technique achieving Dynamic Allocation of Memory has been used. At programming time the programmer just makes use of the tools stored in his tool box.

In the algorithm for achieving controlled image fusion of two images $A$ and $B$, the resulting image is called $\mathrm{C}$. With the aim on improving the quality of image $\mathrm{C}$, this author selects a color $\mathrm{nnn}$ in image A before combining the images, this color will be ignored during fusion. In this case, whenever $A(x, y)$ $=n n n$, the result at pixel $(x, y)$ will simply be $C(x, y)$ $=\mathrm{B}(\mathrm{x}, \mathrm{y})$. Color nnn stands for a RGB triad in strictly color images and for a value between 0 and 255 in grey-leveled images. This addition has improved the fusion results, especially when dealing with color images. Bear in mind that there is no reason to limit this operation to a single value nnn, in some cases it may be convenient to select more than a single nnn to be ignored during fusion. Note that the color to be ignored during the fusion process may also be chosen from image $B$.

In general the steps the algorithm for controlled image fusion must follow are: 

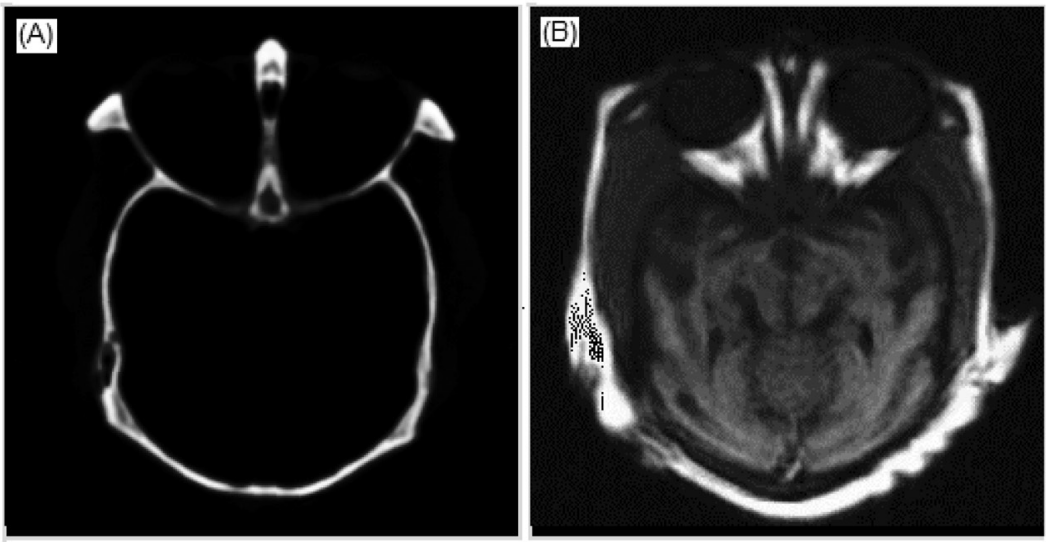

Fig 3

Top: (input) Two medical images $\mathrm{A}$ and $\mathrm{B}$ of the same scene, obtained with different radiations:
(A): Computer tomography
(B): Magnetic resonance

Below: (output) Three different cases of fusion of images $A$ and $B$ :
(1): $50 \%$ and $50 \%$
(2) and (3): $20 \%$ and $80 \%$
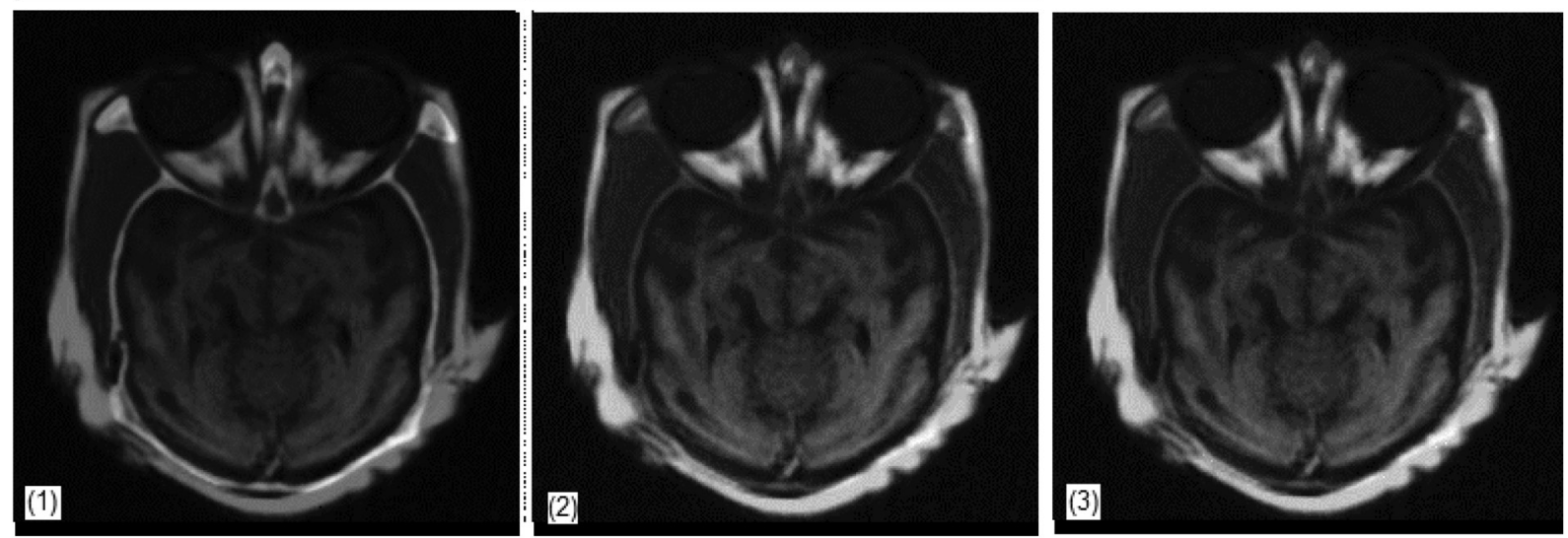

The difference between images (2) and (3) is that in order to generate image (3) a dark color was

ignored during fusion. It can be seen that ignoring a color during fusion improves the image

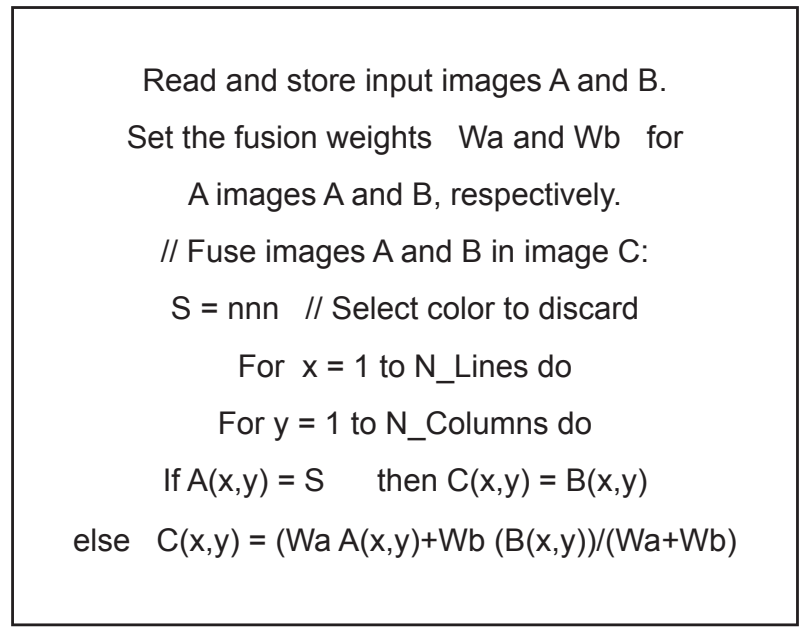

Details on how to read and store the input images $A$ and $B$ are not given because it depends on several factors, one of them being the computer programming language used. Some languages include algorithms that read images in a pixel by pixel mode, others read a whole image line at once. Sometimes the images are stored in memory, other times it is simply loaded onto the screen of the computer monitor. Depending on his experience and objective each programmer must consider his own possibilities.

The algorithm reported here can be easily extended to the fusion of three or more images. In fact this author has already fused three images controlling the contribution of each one to the result.

\section{Experimental results}

Fig 1: At the upper part two images of the same scene are shown, these images have been obtained one in the visible spectrum and the other in the infrared. As it can be appreciated an image provides information not present in the other. Hence individually each image yields a rather low practical benefit. In order to extract useful information from these images, a controlled image fusion of them is carried out. In the lower part of Fig. 1 three different weighted fusions are displayed, the corresponding fusion weights being (a) 50 / 50 (b) $65 / 35$ (c) $75 / 25$

Fig. 2: In the upper part two (input) images of the same scene are shown, these have been obtained with unknown radiations. At the bottom, the fusion 
of the input images with corresponding weights 50 / 50, 30 / 70 and $30 / 70$, are revealed. Notice that output images [2] and [3] have the same fusion weights. With the aim on improving the neatness of output image [3], a dark color in input image A was selected before the fusion, this color does not participate in the fusion and it can be seen that this decision has improved the quality of the output image [3].

Fig 3: At the top two medical images of the same view are displayed. One of these images was obtained by means of Computer Tomography and the other through Magnetic Resonance. The fusion outcome can be seen at the lower part, the fusion proportions being (1) 50 / 50, (2) 20 / 80 and (3) 20 / 80. Once again, before obtaining output image [3] a color in input image A was selected to be ignored during the fusion process.

\section{CONCLUSIONS}

A Virtual Lab to experiment with Controlled Image Fusion by means of the weighted average of the input images has been developed and has been successfully tested. In an attempt to improve the algorithm, the eventual disregard of a pre-selected color in one of the input images has been introduced; this has added quality upgrading to the resulting images, especially when dealing with those of color. The experiments reported here have been applied to the fusion of three color images, with successful results.

The module for Controlled Image Fusion above described has been added to Imagery, the Digital Image Processing Virtual Lab created by the author of this report.

\section{REFERENCES}

[1] Gonzales R, Wintz P. (1987). Digital Image Processing, Addison-Wesley, USA.

[2] Gonzales R, Woods R. (1993). Digital Image Processing (Addison-Wesley, USA.

[3] Gonzales R., Woods R. (2008). Digital Image Processing (Prentice Hall, USA).

[4] Schalkoff R. J. (1989). Digital Image Processing and Computer Vision, Willey, USA.

[5] Jahne B. (1995). Digital Image Processing, Springer-Verlag, USA.

[6] Chen C.H., Pau L. F, Wang P.S.P. (1995). Handbook of Pattern Recognition \& Computer Vision, World Scientific, USA.

[7] Jhane B. (2004). Practical handbook on image processing for scientific and technical applications, CRC Press, USA.

[8] Mitchell, H. B. (2010). Image Fusion: theories, techniques and applications, Springer-Verlag, Germany.

[9] Stathaki, T. (2008). Image Fusion: Algorithms and Applications, Elsevier, The Netherlands.

[10] Blum R.S-Zheng Liu (2006). Multisensor Image Fusion and its Applications, Taylor \& Francis, USA.

[11] Montenegro Joo J. (2009). Imagery 37: Digital Image Processing Virtual Lab., V International Conference on Multimedia and ICT in Education (m-ICTE 2009), Lisboa, Portugal.

[12] Montenegro Joo J. (2011). Virtual Lab for Thresholded Image Difference. Industrial Data, Vol 14, N. ${ }^{\circ}$ 2: 34-37.

[13] Montenegro Joo J. (2012), Thresholded Overlapping of Images, Industrial Data, Vol $15 \mathrm{~N} .{ }^{\circ} 1$. 